\title{
Fenotipos bioquímicos y fagotipos de cepas de Salmonella enteritidis aisladas en Antofagasta, 1997-2000
}

\author{
Juan Silva $A^{a}$, Carmen Aravena $M^{b}$, Jorge Araya $\mathbf{R}^{c}$, \\ Patricia Colque-N avarrod, Inger Kühne, Roland Möllbyf. \\ Biochemical phenotypes and phage \\ types of Salmonella enteritidis strains \\ isolated in Antofagasta during the \\ period 1997-2000
}

Background: PhP-S48 (Phene Plate Techniques AB), a method based on biochemical phenotypes has been developed and used successfully to typify $\mathrm{S}$ enteritidis strains in epidemiological studies. Aim: To identify phenotypes of S enteritidis isolated from eggs, chicken meat and infected humans in Antofagasta during the period 1997-2000. Material and Methods: PhP-S48 and phage typing were used to identify phenotypes of $33 \mathrm{~S}$ enteritidis strains, sixteen isolated from poultry and 17 from clinical sources. S enteritidis ATCC17036 was used as control strain. Results: Twelve biochemical phenotypes (BTs) including 4 common (C) and 8 single (S) were identified. BTs C1 y C3 containing 16 and 5 strains, respectively, accounted for $63.6 \%$ of the isolates. BT C1 was found in poultry and human sources in the period 1997-2000, and BT C3 was isolated from humans, in the period 1999-2000. Using phage typing, 5 phage types (PT) and 3 strains could be not typed (NTs). PT1 and PT21 were the dominant phage types, with 14 and 13 strains respectively. Strains of PT1 were isolated from poultry and human sources in the period 1997-2000. PT21 was found in poultry samples in the period 1997-1998 and in clinical samples, in the period 1997-1998. Combination of biochemical phenotypes and phage typing divided the strains into 5 phenotypes (BT:PT). Two phenotypes were the most frequently isolated, phenotype C1:1 with 8 isolates found in eggs and humans in 1999, and phenotype C1:21 with 5 strains isolated in 19971999. Conclusions: These results indicate the presence of one persistent and one recently emerged phenotype among S enteritidis in Antofagasta, Chile. PhP-S48 also provided information about a relationship among the strains (Rev Méd Chile 2003; 131: 837-45).

(Key-words: Eggs; Poultry products; Salmonella enteritidis; Salmonella phages)

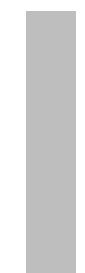

\begin{abstract}
Recibido el 30 de septiembre, 2002. Aceptado en versión corregida el 10 de junio, 2003. Trabajo financiado por proyecto FNDR-3328, Gobierno II Región, Chile y PEI BO11 DINV, Universidad de Antofagasta.

Departamento de Tecnología Médica-INDES, Universidad de Antofagasta, Antofagasta, Chile. Microbiology and Tumorbiology Center, Karolinska Institute, Estocolmo, Suecia.

aTecnólogo Médico, Magíster en Microbiología, PhD en Biología. bioquímico, Magíster en Ciencias Biomédicas (C). 'Tecnólogo Médico, Doctor en Microbiología e Inmunología. d'Ingeniero

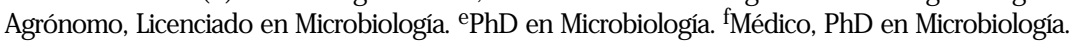

Correspondencia a: Dr. Juan Silva. Departamento Tecnología Médica-INDES. Universidad de Antofagasta. Avenida Coloso s/n. Antofagasta, Chile. Fonofax: (56-55) 637 207. E mail: jsilva@uantof.cl 
$\mathrm{D}$ urante las últimas dos décadas, Salmonella enteritidis ha sido reconocido como uno de los principales agentes etiológicos que causa infecciones gastrointestinales a nivel mundial ${ }^{1,2}$. Estas infecciones ocurren generalmente por el consumo de huevos o productos avícolas contaminados $^{3-5}$. En Sudamérica, los primeros hallazgos en que describieron infecciones por este microorganismo ocurrieron a mediados de la década de 1990-99, originando brotes epidémicos en Argentina, Brasil y Chile ${ }^{6,7}$. En Chile, Senteritidis emergió con características epidémicas en 1994, afectando a un gran número de personas, con tasas que aumentaron en 3.000 por ciento con respecto a años anteriores ${ }^{2}$. Durante el período epidémico, 1994-1996, 90\% de las notificaciones de casos con infecciones por este microorganismo provinieron de Arica y Antofagasta $^{7}$ y gran parte de estas infecciones alimentarias fueron atribuidas al consumo de huevos. Posteriormente, S enteritidis se ha diseminado por todas las regiones de Chile, constituyéndose en un patógeno humano endémico y, por consiguiente, en un importante problema de salud pública ${ }^{8}$.

Diversos autores han tratado de explicar las razones de la emergencia de las infecciones por $\mathrm{S}$ enteritidis en Chile, sugiriendo que los aislamientos de Senteritidis en nuestro país fueron introducidos en los planteles avícolas, por la importación de aves reproductoras de otros países ${ }^{8,9}$.

En los estudios epidemiológicos de S enteritidis se han utilizado diversos marcadores para discriminar acerca de los tipos o clones circulantes, durante los brotes epidémicos. Algunos investigadores sostienen que los métodos genético-moleculares son altamente efectivos, confirmando su enorme potencial para el diagnóstico de estos agentes infecciosos; como es el caso de la electroforesis de campo pulsado (PFGE) ${ }^{10,11}$, la reacción en cadena de la polimerasa (PCR) ${ }^{12,13}$, amplificación randómica de ADN polimórfico $(\text { RAPD PCR })^{14}$ y la ribotipificación ${ }^{15-17}$. Todas estas técnicas presentan diferencias en el grado de discriminación de las cepas y los resultados han sido un poco variables; además, la poca accesibilidad a los laboratorios de hospitales por su elevado costo y manejo de las técnicas, hacen difícil su uso rutinario. Sin embargo, en forma paralela se han estado utilizando algunos marcadores fenotípicos bastante reproducibles para to- dos los laboratorios. Uno de ellos, es la tipificación por bacteriófagos, que permite la separación de las cepas de acuerdo a su patrón de lisis por diferentes virus bacterianos. La fagotipia es un método rápido de tipificación de $\mathrm{S}$ enteritidis y ha sido ampliamente usado en estudios epidemioló$\operatorname{gicos}^{18-20}$.

Estudios de fagotipia realizados en Chile, han demostrado que los fagotipos 1 y 4 de Senteritidis han sido los responsables de la mayoría de las infecciones ocurridas en la reciente epidemia. El fagotipo 1 fue frecuentemente aislado en el norte de Chile (94\%); en cambio, el fagotipo 4 en el centro y sur del país $(92 \%)^{9}$. También, en un estudio orientado a detectar presencia de Senteritidis en productos avícolas en Santiago, se aisló mayoritariamente el fagotipo 4 (59,4\%), concordando con su alto aislamiento en muestras clínicas, y además, se encontró el fagotipo 7 (25\%), el cual no ha sido frecuentemente aislado en infecciones humanas ${ }^{21}$. Estos trabajos, señalan también la importancia de identificar correctamente los tipos de $\mathrm{S}$ enteritidis que están circulando en el país, con el fin de aplicar las medidas de control en forma oportuna.

El sistema Phene-Plate ( $\mathrm{PhP}$ ) es un nuevo método de tipificación fenotípica de serotipos de Salmonella y otras bacterias entéricas, desarrollado en 1985, por Kühn ${ }^{22}$ en Suecia. Es una técnica computarizada y fácil de realizar. El sistema se basa en el análisis numérico de la cinética de 48 reacciones bioquímicas del crecimiento bacteriano, en un medio líquido en una microplaca de titulación. Por cada cepa se produce un set característico de reacciones que se registran, los cuales pueden ser usados para establecer un nivel de identidad entre las cepas bacterianas ensayadas, usando un programa de computación. Para cada serotipo de Salmonella se ha elegido una batería de sustratos específicos con óptima discriminación. El sistema ha sido previamente evaluado por su poder discriminatorio, reproducibilidad $^{23}$ y estabilidad de los marcadores bioquímicos ${ }^{24}$. También ha sido usado exitosamente en estudios epidemiológicos de diferentes serotipos de Salmonella ${ }^{24-26}$.

En base a estos antecedentes, los objetivos de este trabajo fueron: investigar la utilidad del sistema Ph-Plate para determinar los fenotipos bioquímicos de cepas de $\mathrm{S}$ enteritidis $\mathrm{y}$, junto con 
la fagotipia, estudiar la variación de los fenotipos detectados en las cepas de Senteritidis aisladas de productos avícolas y de casos clínicos, durante 1997-2000 en Antofagasta.

\section{MATERIAL y MÉTOdO}

Cepas bacterianas. Un total de 33 cepas de $\mathrm{S}$ enteritidis fueron incluidas en este estudio. Dieciséis fueron aisladas de muestras avícolas, 13 de huevos y 3 de carne de pollo. De las cuales, 10 fueron obtenidas del Instituto de Salud Pública de Chile, que habían sido enviadas por el Laboratorio de Bromatología del Servicio de Salud de Antofagasta y fueron aisladas en 1997-1998. Además, las 6 cepas restantes fueron aisladas en huevos en nuestro laboratorio (1999-2000). También, 17 cepas fueron obtenidas de muestras clínicas de pacientes ambulatorios atendidos por síndrome de diarrea aguda en Consultorios de Atención Primaria de Antofagasta, entre 1999-2000. S enteritidis ATCC 17036 se incluyó como cepa control.

Fagotipificación de las cepas. Las 33 cepas de S enteritidis fueron fagotipificadas de acuerdo con los protocolos estandarizados, previamente descritos por Ward y $\mathrm{col}^{18}$, en el Instituto de Salud Pública de Chile. Los fagotipos fueron abreviados FT.

Fenotipos bioquímicos de las cepas. El sistema Phene-Plate (PhP-S48) se usó para determinar los fenotipos bioquímicos (FTs) de las 33 cepas de S enteritidis y para validar los ensayos, se incluyó la cepa ATCC 17036. El sistema PhP-S48 (Phene Plate $^{\mathrm{TM}}$ System $\mathrm{AB}$, Estocolmo, Suecia) consiste en microplacas que contienen 2 sets paralelos de 48 substratos desecados, especialmente elegidos para los fenotipos bioquímicos de cepas de Salmonella de diferentes serotipos. Para cada serotipo un set de pruebas con óptimo grado de discriminación ha sido seleccionado y anexado a un programa de computación (PhPWIN), que permite realizar los cálculos requeridos. Los sustratos utilizados para la tipificación de las cepas de $\mathrm{S}$ enteritidis, se muestran en la Tabla 1.

Una colonia de la cepa a ensayar fue sembrada en tubo con $10 \mathrm{ml}$ de proteosa peptona (Difco) $0,1 \%$, conteniendo azul de bromotimol $0,01 \%$ y se inocularon alícuotas de $150 \mu \mathrm{l}$ a los pocillos de las microplacas pre-preparadas, conteniendo los

Tabla 1. Set de reactivos usados para determinar fenotipos bioquímicos de Salmonella enteritidis por el Sistema Ph-Plate

\begin{tabular}{|rlllll|}
\hline Test & Reactivo & Test & Reactivo & Test & Reactivo \\
\hline 1 & Acido Manoico & 17 & Adonitol & 33 & Arbutina \\
& -d-lactona & & & & \\
2 & L-Arabinosa & 18 & Inositol & 34 & B-Metil glucósido \\
3 & D-Xilosa & 19 & D-arabitol & 35 & 5-Ketogluconato \\
4 & Galactosa & 20 & Glicerol & 36 & Gluconato \\
5 & Maltosa & 21 & Maltitol & 37 & Melobionato \\
6 & Celobiosa & 22 & Sorbitol & 38 & Galactolactona \\
7 & Trehalosa & 23 & Dulcitol & 39 & Salicina \\
8 & Palatinosa & 24 & pH 7,4 Control & 40 & pH 5,5 Control \\
9 & Sucrosa & 25 & Sorbosa & 41 & Citrato \\
10 & Lactosa & 26 & Deoxiglucosa & 42 & Fumarato \\
11 & Melibiosa & 27 & Deoxiribosa & 43 & Malinato \\
12 & Lactulosa & 28 & Ramnosa & 44 & Malonato \\
13 & Gentobiosa & 29 & D-Fucosa & 45 & Piruvato \\
14 & Melezitosa & 30 & L-Fucosa & 46 & L-Tartarato \\
15 & Rafinosa & 31 & Tagatosa & 47 & Urea \\
16 & Inopina & 32 & Amigdalina & 48 & Ornitina \\
\hline
\end{tabular}


substratos $(3,75 \mathrm{~g} / \mathrm{l})$ en cada pocillo. Los inóculos se dispusieron con micropipetas múltiples y para conseguir una apropiada rehidratación de los substratos, las placas inoculadas fueron mantenidas refrigeradas a $4^{\circ} \mathrm{C}$ toda la noche y, posteriormente, incubadas a $37^{\circ} \mathrm{C}$. Después de la incubación, las placas fueron leídas en un lector de microplacas con un espectrofotómetro (iEMS, Labsystems, Finland) a $620 \mathrm{~nm}$, la absorbancia de cada reacción fue medida a las 7, 24 y 48 h de incubación.

Los valores de absorbancia, transferidos a un computador personal, fueron multiplicados por 10, así los resultados producidos estuvieron en un rango de 0 a 30 por cada reacción; valores bajos indicaban reacciones ácidas (amarillo) y valores altos reacciones alcalinas (azul profundo). Después de la lectura final, el valor promedio de las 3 lecturas fue calculado proveyendo 12 números diferentes que varían 0-30 por cada cepa, constituyendo el fingerprinting bioquímico para cada cepa. Similitudes entre las cepas ensayadas fueron calculadas como coeficientes de correlación (r) de acuerdo con trabajos previos ${ }^{23}$ y fueron reunidas de acuerdo al método de grupo par no pesados con promedios aritméticos (UPGMA) ${ }^{27}$ en un dendrograma. Las cepas que mostraron coeficientes de correlación más altos que el nivel de identidad usado por el sistema PhP-S48 $(0,980)$ fueron asignados al mismo fenotipo bioquímico ${ }^{28}$. Los fenotipos bioquímicos que se encontraron en más de una cepa fueron llamados fenotipos comunes (C), y aquellos que presentaron solamente un aislamiento fueron llamados fenotipos sólos (S).

La recolección de los datos, incluyendo las lecturas ópticas, fueron realizadas con el software Programa PhPWIN (PhP-Plate Systems AB).

\section{Resultados}

El sistema Ph-Plate de tipificación permitió diferenciar 12 fenotipos bioquímicos (FB) (Tabla 2). De ellos, 4 fueron FB comunes (C) y 8 FB solos (S) y la cepa ATCC 17036 presentó en este caso un FB solo (S). Los FBs C1 y C3 contenían 16 y 5 cepas respectivamente, lo que corresponde a $63,6 \%$ del total de las cepas ensayadas. El sistema Ph-Plate permitió establecer que 11 cepas de origen avícola y 5 clínicas, pertenecían al FB C1. Además en el FB C1, se encontró que 5 cepas avícolas y 5 clínicas fueron aisladas en el año 1999, las restantes cepas ambientales fueron aisladas en 1997 (3 cepas) y 1998 (3 cepas). En cambio, las 5 cepas del FB C3 fueron aisladas de muestras clínicas, aisladas en los años 1999 (2 cepas) y 2000 (3 cepas). El FB C2 incluye 2 cepas clínicas, aisladas en 1999 y 2000; y Tabla 2. Fenotipos bioquímicos (FB) de cepas de Salmonella enteritidis aisladas
de muestras avícolas y clínicas en Antofagasta. C hile. 1997-2000

\begin{tabular}{|cccc|}
\hline $\begin{array}{c}\text { Fenotipos } \\
\text { (FB) }\end{array}$ & $\begin{array}{c}\text { Número } \\
\text { de cepas }\end{array}$ & \multicolumn{2}{c|}{ Origen y año aislamiento de cepas } \\
Avícola & Clínico \\
\hline C-1 & 16 & $1997(3)-1998(3)-1999(5)$ & $1999(5)$ \\
C-2 & 2 & - & $1999(1)-2000(1)$ \\
C-3 & 5 & - & $1999(2)-2000(3)$ \\
C-4 & 2 & $1998(1)$ & $1999(1)$ \\
S-1 & 1 & - & $2000(1)$ \\
S-2 & 1 & $1999(1)$ & - \\
S-3 & 1 & $1997(1)$ & - \\
S-5 & 1 & $1998(1)$ & - \\
S-6 & 1 & - & $2000(1)$ \\
S-7 & 1 & - & $1999(1)$ \\
S-8 & 1 & - & - \\
S-9 & 1 & $1998(1)$ & 17 \\
Total & 33 & 16 & \\
\hline
\end{tabular}


Tabla 3. Fagotipos (FT) de cepas de Salmonella enteritidis aisladas de muestras avícolas y clínicas en Antofagasta. 1997-2000

\begin{tabular}{|cccc|}
\hline $\begin{array}{c}\text { Fagotipos } \\
\text { (FT) }\end{array}$ & $\begin{array}{c}\mathrm{N} \text { de } \\
\text { cepas }\end{array}$ & \multicolumn{2}{c|}{ Origen y año aislamiento de cepas } \\
Avícola & Clínico \\
\hline 1 & 14 & $1997(1)-1999(6)$ & $1999(2)-2000(5)$ \\
3 & 1 & $1998(1)$ & - \\
4 & 1 & - & $1999(1)$ \\
7 & 1 & $1997(1)$ & - \\
21 & 13 & $1997(2)-1998(5)$ & $1999(5)-2000(1)$ \\
NT & 3 & - & $1999(2)-2000(1)$ \\
Total & 33 & 16 & 17 \\
\hline
\end{tabular}

C4 presentó 1 cepa ambiental (1998) y 1 cepa clínica (1999). Las 8 cepas pertenecientes a los FBs (S) fueron todas aisladas de muestras avícolas (4) y clínicas (4), en los años 1997 a 2000. Estos resultados fueron validados con la cepa ATTC 17036 de S enteritidis.

Los estudios de fagotipia de las 33 cepas de S enteritidis permitió caracterizar 6 fagotipos, incluyendo aquellos designados como no tipificables (NT), los cuales se presentan en la Tabla 3. Los dos FTs dominantes fueron: FT1 con 14 cepas de S enteritidis y FT21 con 13 cepas, lo que incluye el $81,8 \%$ de las cepas ensayadas. Hubo 3 cepas que no pudieron ser asignadas a ningún fagotipo reconocido y fueron consideradas como no tipificables (NT). De las cepas del FT1, 7 de ellas fueron aisladas de muestras avícolas, 1997 (1 cepa) y 1999 (6), y además, 7 de muestras clínicas 1999 (2) y 2000 (5). Las cepas encontradas en el FT21, 7 fueron de muestras avícolas, 1997 (2 cepas) y 1998 (5), y además, seis de muestras clínicas, 1999 (5 cepas) y 2000 (1 cepa). Los aislamientos de las cepas clínicas y avícolas del FT21 ocurrieron en distintos años, como para establecer una relación entre ellas. En este trabajo, no se incluyeron cepas clínicas aisladas en el período 1997-1998, como así también, cepas avícolas pertenecientes al FT21 en 1999-2000. Las cepas del FT4 y NT fueron aisladas de muestras clínicas, en los años 1999 y 2000; y las de los FT3 y FT7 pertenecieron a muestras avícolas 1998 y 1997, en cada caso.

El sistema Ph-Plate permitió reconocer diferentes fenotipos y además, mediante el Programa
Computacional PhPWIN estableció las similitudes entre las cepas ensayadas que presentaron un mayor coeficiente de correlación $(0,980)$. Sus resultados fueron registrados en un dendrograma, que permitió la comparación de los dos sistemas de tipificación (Figura 1). Al combinar los fenotipos bioquímicos con los fagotipos (FB:FT), pertenecientes a una misma cepa de $\mathrm{S}$ enteritidis, se pudieron encontrar 6 fenotipos comunes (con más de una cepa) y 13 solos (FB:FT). Entre los tipos comunes, dos mayores fenotipos fueron identificados. La combinación del fenotipo bioquímico y fagotipo $\mathrm{C} 1: 1$ presentó el mayor número de aislamientos (8 cepas), de las cuales 5 correspondieron a cepas avícolas aisladas de huevos en 1999 y 3 a cepas clínicas aisladas en 1997 (1) y 1999 (2). También, la combinación de ambos fenotipos más frecuente fue C1:21 con 5 aislamientos, encontrándose 1 cepa aislada en huevo en 1997, dos cepas de huevos en 1998, una de carne de pollo en 1998 y una de muestra clínica en 1999. En los otros fenotipos comunes encontrados (FB:FT), los aislamientos correspondieron a 2 cepas en cada caso y presentaron variaciones en los años.

\section{DisCUSIÓN}

En los últimos 20 años, las infecciones por diferentes serotipos de Salmonella han aumentado notablemente en todo el mundo, especialmente infecciones gastrointestinales causadas por $\mathrm{S}$ enteritidis $^{1}$. En diversos reportes, se ha señalado la necesidad de contar con buenos marcadores 


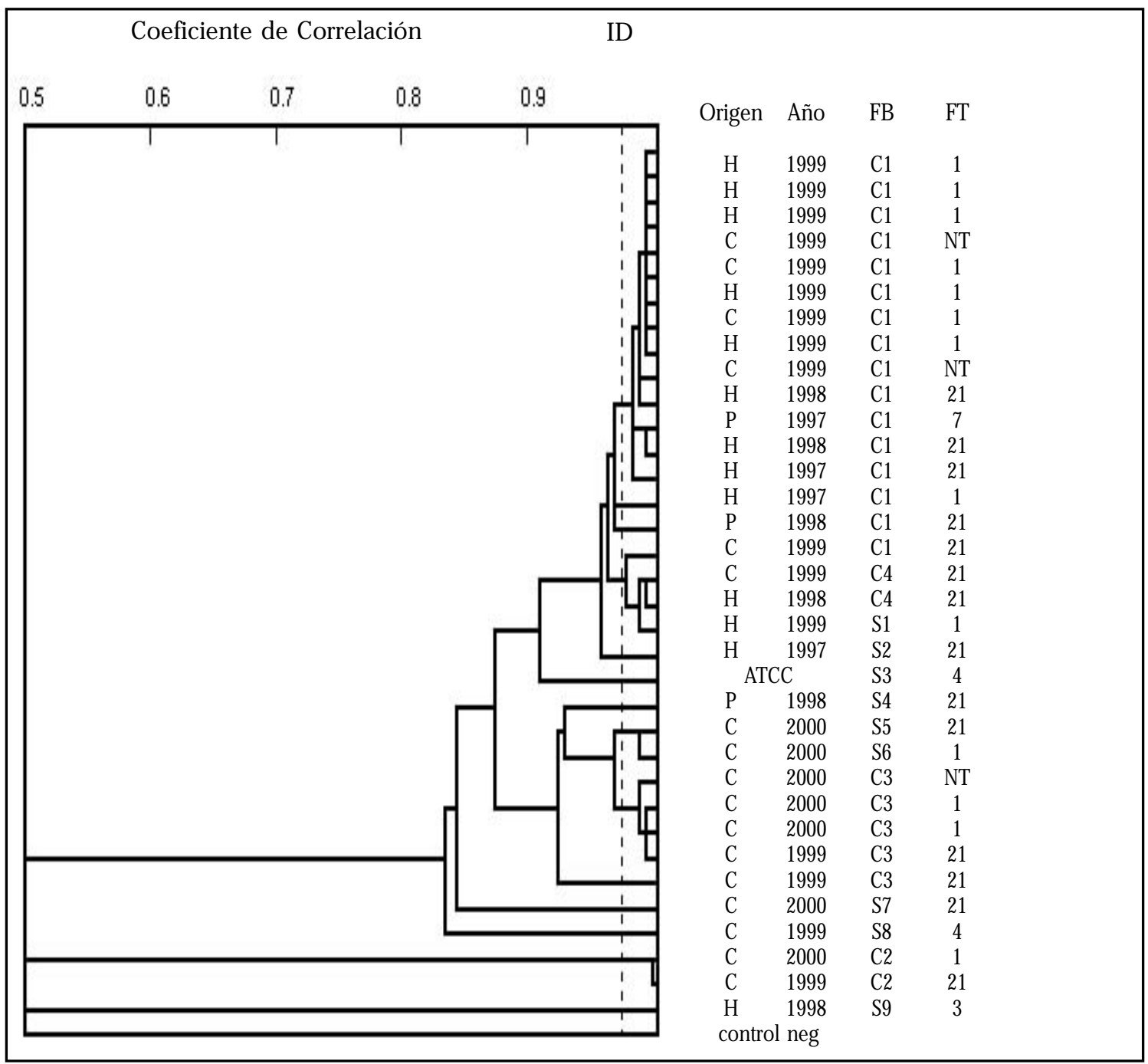

Figura 1. Dendrograma presentando las variaciones entre fenotipos bioquímicos (FBs) y fagotipos (FTs) de 33 cepas de Salmonella enteritidis aisladas en Antofagasta, entre 1997 y 2000. El nivel de identidad (ID) señala que el nivel de identidad elegido $(\mathrm{r}=0,980)$ para cada cepa asignada al mismo FB. NT, cepas no tipificables. $\mathrm{H}=$ huevos; $\mathrm{P}=$ carne de pollo y $\mathrm{C}=$ clínicas.

epidemiológicos, ya que la serotipificación por sí sola no es efectiva como marcador. Diversas técnicas han sido empleadas para este fin, entre ellas sobresalen la fagotipificación ${ }^{18}$ que conjuntamente con análisis del perfil plasmidial ${ }^{29,30}$ y los patrones de resistencia (antibiotipo) ${ }^{31}$ han sido usadas extensamente para tipificar cepas de S enteritidis. También, marcadores bioquímicos han sido usados para tipificar los diferentes serotipos de Salmonella, pero en el caso de S enteritidis existe escasa información sobre los datos obtenidos. Katouli y $\operatorname{cols}^{26}$ realizaron un estudio para determinar la variación de fenotipos bioquímicos y fagotipos de cepas de S enteritidis, utilizando el sistema Ph-Plate Systems y la fagotipia. Los resultados descritos demostraron que este método presenta excelentes ventajas para tipificar cepas de $\mathrm{S}$ enteritidis. Ya que en un total de 86 cepas clínicas ensayadas, 56 cepas fueron reconocidas en dos FB dominantes y correspondieron casi al 
$65 \%$ del total de los aislamientos. Al combinar el fenotipo bioquímico (FB) con el fagotipo (FT) detectaron 10 fenotipos comunes (FB:FT) en las cepas de $\mathrm{S}$ enteritidis y en uno de los fenotipos dominantes, 9 aislados fueron identificados por el mismo FB y FT. Al igual que en nuestro trabajo, se incluyó como control la cepa de $\mathrm{S}$ enteritidis (ATCC 17036) que presentó las características bioquímicas correspondientes al mismo serotipo.

En este trabajo, también se utilizó el sistema Ph-Plate que permitió la identificación de fenotipos bioquímicos de cepas $\mathrm{S}$ enteritidis aisladas de muestras clínicas y avícolas de Antofagasta. Los resultados obtenidos fueron altamente satisfactorios. De un total de 33 cepas de $\mathrm{S}$ enteritidis ensayadas, se logró identificar 12 FB con este método. Dos tipos FB dominantes fueron encontrados, encontrándose 22 cepas $(63,6 \%$ del total de las cepas) y en 13 de ellas, hubo correspondencia con los fagotipos mayormente aislados.

La fagotipia de las cepas de S enteritidis reveló la presencia de dos tipos dominantes de fagotipos, el FT1 (14 cepas) y FT21 (13 cepas). En estudio realizado con cepas chilenas, colectadas entre 1975 y 1996 por el Instituto de Salud Pública de Chile, se encontró que el FT1 era frecuentemente aislado en el norte y el FT4 en el centro y sur del país ${ }^{9}$. Estos resultados concuerdan en parte con nuestro estudio, ya que el fagotipo 1 aparece como el más común FT y se aisla con gran frecuencia en infecciones humanas por consumo de productos avícolas contaminados, desde 1997 al 2000. También, ha sido reconocido como el FT prevalente en infecciones ocurridas en el norte de Chile ${ }^{9}$, en la epidemia del 94-96. Todos estos trabajos señalan la existencia del FT1, como un fagotipo persistente en el norte de Chile. El FT1 es frecuentemente aislado en Europa ${ }^{9}$ y es posible que haya sido introducido a estas regiones del país en aves reproductoras provenientes de dicho continente.

Sin embargo, desde 1997 otro fagotipo aparece en nuestro escenario y es el FT21 (13 aislados), el cual había sido descrito en Santiago solamente en 1 caso en una muestra de productos avícolas ${ }^{21}$. En nuestro estudio, se aislaron 7 cepas de productos avícolas y 6 de muestras clínicas, pero ambos en diferentes períodos. La frecuencia de aislamiento del FT21, puede deberse a la importación de nuevas aves reproductoras de otros lugares para disminuir la presencia de $\mathrm{S}$ enteritidis en las granjas avícolas, pero desgraciadamente estas aves al parecer venían contaminadas con el FT21. El FT21 ha sido frecuentemente aislado en la República Checa, Austria e Inglaterra ${ }^{32}$. Este hallazgo puede significar que tenemos un nuevo fagotipo emergente en el norte de Chile, FT21. En nuestro estudio, el FT21 fue detectado en los años que se analizaron muestras de huevos y pollos (1997 y 1998) y en muestras clínicas (1999 y 2000). Esta intermitencia en los hallazgos de este fagotipo, se debe a que en un principio se planeó el estudio con cepas avícolas y clínicas aisladas en 1999-2000. Pero, en los estudios de muestras avícolas se aislaron solamente 6 cepas de S enteritidis en huevos expendidos en Antofagasta (1999-2000) (resultados no publicados). Debido a su escaso número, se solicitaron cepas avícolas al Servicio de Salud de Antofagasta, pero solamente se habían aislado 10 cepas de productos avícolas en 1997-1998 y se incluyeron en el estudio. Por ello, no se puede establecer la relación entre en ellos y la vía de transmisión. Sin embargo, a pesar de que el tamaño de muestra es pequeño es posible que pudieran haber estado presentes en muestras avícolas y clínicas en el mismo período, al examinar un mayor número de muestras. Sólo podemos señalar que la presencia del FT21 en productos avícolas y muestras clínicas, sugiere una vía de transmisión al ser humano por el consumo de estos productos avícolas contaminados con este fagotipo.

Al comparar la relación FB:FT, se encontraron que los fenotipos más comunes fueron $\mathrm{C} 1: 1 \mathrm{y}$ C1:21, con 8 y 5 aislamientos, respectivamente. Las cepas dominantes fueron aisladas de huevos (7), carnes de pollo (1) y clínicas (5), sugiriendo una estrecha relación y un origen clonal común. Esto concuerda con lo descrito por otros autores ${ }^{3-20}$, en el sentido, que la vía de la transmisión de $\mathrm{S}$ enteritidis a los humanos en la zona norte de Chile, puede ser a través del consumo de productos avícolas contaminados con los mismos tipos de $\mathrm{S}$ enteritidis. También, la combinación de los fenotipos bioquímicos y fagotipos indicaron que solamente 8/14 del FT1 estaba presente en un mismo FB (C1). Las 6 cepas restantes pertenecían a otros FB (C2 y C3). El encontrar 8 cepas pertenecientes a un mismo fenotipo bacteriano que ha sido discriminado por ambos métodos fenotípicos (sistema $\mathrm{Ph}$ Plate y fagotipia), nuevamente refuerzan la idea de 
la vía de transmisión de un mismo clon, es a través del consumo de productos avícolas contaminados.

$\mathrm{Al}$ examinar la variación a través de los años de aislamiento de los fenotipos de S enteritidis, el sistema Ph-P-S48 con sus fenotipos bioquímicos permite explicar en mejor forma la diseminación de las cepas de S enteritidis en Antofagasta. En el año 1999, el FB C1 fue aislado de 5 muestras de productos avícolas y 5 de clínicas. Las demás cepas del FB C1 fueron aisladas en los productos avícolas, en años anteriores, en los cuales no se recolectaron cepas de origen clínico y es posible, que también estuvieran presentes. En relación a los años, muestra una clara persistencia en su aislamiento durante todos los años. En cambio, la fagotipia de las cepas de Senteritidis, mostró que el FT1 en los productos avícolas fue aislado en el año 1997 (1 aislado) y 1999 (6 aislados), y en las muestras clínicas en 1999 (2 aislados) y 2000 (5 aislados), mostrando variaciones en los aislamientos durante los años de estudio. También, el FT1 presentó diversos fenotipos bioquímicos detectados por el sistema de PhP-S48, lo que indica que este método es mucho más discrimatorio en la

\section{REFERENCIAS}

1. Rodrígue DC, Cameron DN, Phur ND, Brenner FW, St Louis ME, Wachsmuth KI, TauXe RV. Comparison of plasmid profiles, phage types and antimicrobial resistance patterns of Salmonella enteritidis isolates in United States. J Clin Microbiol 1992; 30: 854-7.

2. Silva J. Salmonella enteritidis un patógeno emergente de origen aviario. Rev Cs Salud 2000; 4: 25-36.

3. Centers for Disease Control and Prevention. Outbreaks of Salmonella serotype enteritidis infection associated with eating raw or undercooked shell eggs-United State, 1996-1998. Morb Mortal Wkly Rep 2000; 49: 73-9.

4. Henzler DJ, Ebel E, Sanders J, Kradel D, Mason J. Salmonella enteritidis in eggs from commercial chicken layer flock implicated in human outbreaks. Avian Dis 1994; 38: 37-43.

5. Mishu B, Griffin PM, Tauxe R, Cameron D, HUTHCHESON RH, ACHAFFNER W. Salmonella enteritidis gastroenteritis transmitted by intact chicken eggs. Ann Intern Med 1991; 115: 190-4. identificación de los fenotipos de S enteritidis, lo cual ha sido reconocido en trabajos anteriores ${ }^{26}$.

Finalmente, estos resultados muestran la presencia de dos mayores fenotipos (FB:FT) entre las cepas de S enteritidis aisladas entre 1997-2000 en Antofagasta. El fenotipo C1:1, el cual fue el más común entre las cepas aisladas durante 1997-1999, indicando una persistencia de este particular fenotipo a través del tiempo en Antofagasta. Otro fenotipo, representado por un nuevo tipo clonal emergente y diseminado (C1:21), que contiene cepas aisladas en 1997-1999. También, se concluye que la determinación de fenotipos bioquímicos por el método Ph-Plate, es fácil de realizar y constituye una nueva herramienta de tipificación bacteriana que se debe incorporar en estudios epidemiológicos de cepas de S enteritidis y de otras bacterias entéricas en nuestro país. Además, no sólo es una buena técnica que puede detectar cepas idénticas, sino que también, permite establecer la relación entre ellas. Esta información puede ser utilizada para trazar el origen de las cepas patógenas en estudios epidemiológicos.

6. Irino K, Fernnandes SA, Tavecchi AT, Neves BC, Díaz AM. Progression of Salmonella enteritidis paghe type 4 strains in Sao Paulo State. Rev Inst Med Trop Sao Paulo 1996; 38: 193-6.

7. Fica A, Fernández, A, Prat P, Figueroa O, Gamboa R, TsuneKaWA I, Heitman I. Salmonella enteritidis, un patógeno emergente en Chile. Rev Méd Chile 1997; 125: 544-51.

8. Fica A, Alexandre M, Prat S, Fernández A, FernánDez O, HeitTMann G. Cambios epidemiológicos de las salmonelosis en Chile. Desde Salmonella typhi a Salmonella enteritidis. Rev Chil Infect 2001; 18: 85-93.

9. Prat S, Fernández A, Fica A, Fernández J, Alexandre M, Heitmann G. Tipificación fágica de aislados de Salmonella enteritidis de muestras clínicas, alimentarias y avícolas en Chile. Rev Panam Salud Pública 2001; 9: 7-12.

10. Suzuki $Y$, Ishihara M, Matsumoto M, Arakawa S, Saito M, Ishikawa et al. Molecular epidemiology of Salmonella enteritidis. An outbreak and sporadic cases studied by means of pulsedfield gel electrophoresis. J Infection 1995; 31: 211-7. 
11. Powell NG, Threlfall EJ, Chart $\mathrm{H}$, Rowe B. Subdivision of Salmonella enteritidis PT4 by pulsed-field gel electrophoresis: potential for epidemiological surveillance. FEMS Microbiol Lett 1994; 119: 193-8.

12. Fadl AA, NguYen AV, Khan MI. Analysis of Salmonella enteritidis isolated arbitrarily primer PCR. J Clin Microbiol 1995; 33: 987-9.

13. López-Molina N, Laconcha I, Rementeria A, Audicana A, Perales I, Garaizar J. Typing of Salmonella enteritidis of different phage types of PCR fingerprinting. J Appl Microbiol 1998; 84: 877-82.

14. Soto SM, Guerra B, GonzÁlez-Hevia MA, Mendoza MC. Potential of three way randomly amplified polymorphic DNA analysis as a typing method for twelve Salmonella serotypes. Appl Environ Microbiol 1999; 65: 4830-6.

15. Esteban E, Snipes K, Hird D, Kasten RY, Kinde H. Use of ribotyping for characterization of Salmonella serotypes. J Clin Microbiol 1993; 31: 233-7.

16. Olsen JE, Skov MN, Threifall EJ, Brown DJ. Clonal lines of Salmonella enteritidis documented by IS200-, ribo-, pulsed-field gel electrophoresis and RFLP typing. J Med Microbiol 1994; 40: 15-22.

17. Thong KL, Ngeow Y, Altwegg M, Navaratman PY, PANG T. Molecular analysis of Salmonella enteritidis by pulsed-field gel electrophoresis and ribotyping. J Clin Microbiol 1995; 33: 1070-4.

18. WARD L, DESA RJH, RowE B. A phage-typing scheme for Salmonella enteritidis. Epidemiol Infect 1987; 99: 291-4.

19. HickMAn-Brenner FW, Stubbs AD, FARMer JJ $3^{\text {rD }}$. Phage typing of Salmonella enteritidis in the United States. J Clin Microbiol 1991; 29: 2817-23.

20. Evans MR, Lane W, Ribeiro CD. Salmonella enteritidis PT6: another egg associated salmonellosis? Emerging Infect Dis 1998; 4: 667-9.

21. Aiexandre M, Pozo C, González V, Martínez MC, Prat S, Fernández A, Fica A, Fernández O, Heitmann G. Tipificación fágica de aislados de Salmonella enteritidis de muestras clínicas, alimentarias y avícolas en Chile. Rev Méd Chile 2000; 128: 1075-83.

22. KüHN I. Biochemical fingerprinting of Escherichia coli: a simple method for epidemiological investigations. J Microbiol Med 1985; 3: 159-70.

23. KüHN I, BURMAN LG, ERIKSSON L, MöшBY R. Subtyping of Klebsiella by biochemical fingerprinting: a simple system for epidemiological investigations. J Microbiol Methods 1990; 11: 177-85.
24. Katoul M, Wown R, Kühn I, Farhoudi-Moghaddam AA, MöшвY R. The use of biochemical fingerprinting, phage typing and antimicrobial susceptibility testing in detection of epidemic strains of Salmonella of serotype Typhimurium in Iran. J Med Microbiol 1992; 37: 252-7.

25. Katoul M, Kühn I, Brauner A, FarhoudiMoghaddam AA, Mölby R. Application of biochemical fingerprinting to the investigation of clonal group of Salmonella of serotype Havana. J Med Microbiol 1992; 36: 382-8.

26. Katoul M, Seufrer RH, Wown R, KüHn I, Mö山by R. Variations in biochemical phenotypes and phage types of Salmonella enteritidis in Germany 198092. Epidemiol Infect 1993; 111: 199-207.

27. SNeath PHA, Sokal RR. Nuneral Taxonomy. WH: Freemam, San Francisco, California 1973.

28. Kühn L, Aшestam G, Stenström TA, Möшby R. Biochemical fingerprinting of water coliform bacteria, a new method for measuring the phenotypic diversity and for comparing different bacterial populations. Appl Environ Microbiol 1991; 57: 3171-7.

29. Stubbs AD, Hickman Brenner FW, CAMERon DN, FARMER JJ. Differentiation of Salmonella enteritidis phage type- 8 strain, evaluation of 3 additional phage typing systems, plasmid profiles, antibiotic susceptibility patterns and biotyping. J Clin Microbiol 1994; 32: 199-201.

30. Wachsmuth IK, Kiehlbauch JA, Bopp CA, Cameron DN, Strockbine NA, Wells JG et al. The use of plasmid profiles and nucleic acid probes in epidemiologic investigations of foodborne, diarrheal diseases. Int J Food Microbiol 1991; 12: 77-89.

31. Ling JM, Koo IC, Kam KM, Cheng AF. Antimicrobial susceptibilities and molecular epidemiology of Salmonella enterica serotype enteritidis strains isolated in Hong Kong from 1986 to 1996. J Clin Microbiol 1998; 36: 1693-9.

32. Rychuk I, Svestrova A, Karpiskova R. Subdivision of Salmonella enterica serovar enteritidis phage types PT14b y PT21 by plasmid profiling. Vet Microbiol 2000; 74: 217-25.

Agradecimientos

Nuestros agradecimientos al Servicio de Salud de Antofagasta y al Laboratorio «arita Núñez» de la Corporación Municipal de Antofagasta por facilitamos las cepas de Senteritidis incluidas en este estudio. 